\title{
Integrating Subsurface Core and Log Data to Model Tar Mat in a Carbonate Upper Jurassic Reservoir in Offshore Abu Dhabi - UAE
}

\author{
Khalil Al Hossani, Mohamed. I Sayed \& T. M. Matarid (Abu Dhabi Marine Operating Company \\ (ADMA-OPCO)
}

\begin{abstract}
Tar Mats are considered as heavy, rich in Asphaltene, and unmovable hydrocarbon that may exist with oil reservoirs. Mostly, they lay at the bottom of the oil column isolating the oil reservoirs from aquifers. Tar Mats are commonly observed in many oil fields in the Middle East, either as continuous surface or as patchy isolated bodies within the oil reservoirs. However, they represent a volume of a non-producible hydrocarbon-in-place.
\end{abstract}

This paper presents an interpretation, evaluation and modeling a Tar Mat within an Upper Jurassic Reservoir, Offshore Abu Dhabi. The Tar Mat was observed in 6 cored wells out of 9 drilled wells in a Green Field. There were many challenges encountered during the evaluation and modeling the Tar Mat outlined as follows: -

1. Top of Tar Mat was interpreted at different levels within the targeted reservoir.

2. Limited number of drilled wells in the field

3. Some wells didn't penetrate the full reservoir to evaluate "measure" the Tar Mat thickness.

4. Absence of hydraulic data "Green Field".

Core data and available logs were integrated \& provided valuable results to interpret the top of the Tar Mat. In addition, a good quality 3D seismic data was used to generate porosity volume that assisted to interpret the negative porosity values as an extension of Tar Mat between wells.

Tar mat has a big impact on the oil field development and well placement scheme especially when it is present as a continuous surface. The water injection wells should be carefully allocated above the Tar Mat seeking the best pressure support.

In the static 3D geological model, Tar Mat was generated as a bottom limit for the oil reservoir replacing the OWC. Also, multi-realization modeling option was used to model the Tar Mat as patchy isolated bodies as part of reservoir uncertainty evaluation. It was considered as an inactive interval in the dynamic model keeping chances to update it as an active in case of considering Tar Mat as an isolated bodies. Zero transmissibility was considered in the model in all directions for more flexibility of Tar Mat volume sensitivities or further development options as will be viable in the future. 


\section{Introduction}

The studied field is a green field located offshore Abu Dhabi (UAE). Nine wells were penetrated the late Jurassic reservoirs and only six were cored with poor lateral distribution. Four major Jurassic reservoirs occur in the studied field, with wide range of uncertainty. A detailed reservoir characterization study was conducted on the Jurassic reservoir as part of the preparation for Full Field Development Plan (FFDP). During the study, many uncertainties were addressed related to structure, reservoirs quality, reservoirs strategraphy, fluid characterization, Tar Mat origin and distribution...etc.

This study will focus on one major reservoir that includes about $40 \%$ of the reserves and has big development challenges due to the presence of Tar Mat.

A FFDP was proposed for this field considering the Tar Mat as a continuous surface covering the entire field at the targeted reservoir level. Many challenges raised during the evaluation of the FFDP scenarios related to Tar Mat distribution (lateral and vertical), Tar Mat origin, and relationship between Tar Mat precipitation and reservoir facies and OWC.

\section{Tar Mat Observation and Evaluation}

The Tar Mat was physically observed in six cored wells during the detailed sedimentlogical study that completed in year 2008. It was observed as a sharp contact overlaid with hydrocarbon column in some wells and as discontinuous patches distributed within facies. During the study, Tar Mat was observed at different vertical levels within the reservoir and hasn't any relation to the facies distribution and reservoir quality (texture).

On the other hand, it was noticed that the Tar Mat in the northern flank of the field was picked near top of the reservoir, while it was precipitated deeper in southern flank. Also, the picked top tar mat in the eastern flank hasn't any relation to the top of Tar Mat in north and south flanks. This information raised a concern about the relationship between Tar Mat and structure growth. The thickness of Tar Mat is still uncertain because only 2 wells only penetrated the whole reservoir thickness while the rest were targeting the oil column and avoiding the non-reservoir interval of the Jurassic reservoir.

Later, the Tar Mat tops that picked from cores were interpreted to log data in order to define the top Tar Mat in the non-cored wells. The main observation was that the NPHI and RHOP logs values drops dramatically with presence of Tar Mat. This was very helpful to identify the sharp contact of top Tar Mat form logs.

The most recent 3D seismic data was integrated with both core and log data seeking better understanding and more controlling the lateral distribution of Tar Mat. Top Tar Mat form log and core data were integrated with the porosity prediction from 3D seismic data. This technique showed an agreement between picked top Tar Mat and 3D seismic data that leaded to interpret it as continuous surface over entire field.

The interpreted Tar Mat surface is still uncertain due to lack of data related to the bottom of Tar Mat. This could lead to many scenarios related to hydrocarbons bellow the Tar Mat and could increase the OOIP of the field. 


\section{Tar Mat Modeling}

The interpreted Tar Mat surface was imported into the 3D geological modeling software and adjusted to well data. in this case the Tar Mat surface was considered as the bottom limit of the oil column and replaced the oil water contact. Multi-realization modeling option was applied on Tar Mat to investigate the vertical variation uncertainty between wells.

On the other hand, the identified top Tar Mat tops from core and log data were imported in the modeling software as discrete files similar to the rock type data. Then it was modeled using facies and rock types modeling options to generate isolated Tar Mat bodies within the targeted reservoir. Different lateral extend values were used at each well location and the vertical proportion curve controlled the vertical distribution of the Tar Mat. Modeling Patchy Tar Mat was part of an uncertainty study and wasn't used to set the FFDP.

\section{Field Development Scenario}

The Tar Mat was represented in the model as active cells with zero transmissibility. It's proposed to act as a barrier between the oil pool and the water aquifer. So, the aquifer doesn't supply and pressure support to the reservoir (Figure 1).

Peripheral water injection was considered to develop the reservoir. The water injectors planned to be allocated above the Tar Mat surface and within the oil column to compensate the absence of the aquifer pressure support. The Tar Mat surface in the peripheral area covers the lower reservoir quality in the southern flank. In the northern flank, Tar Mat filled the upper part of the reservoir (near top) and blocked the pores of the good reservoir facies. This leaded to avoid placing water injectors in this area to avoid any early water breakthrough.

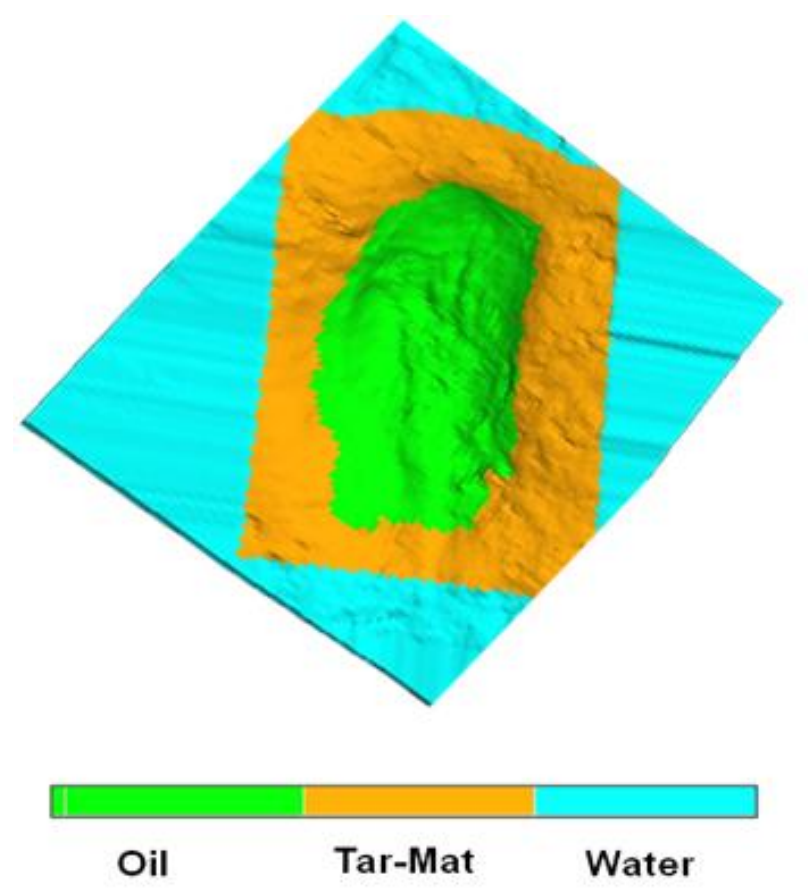

